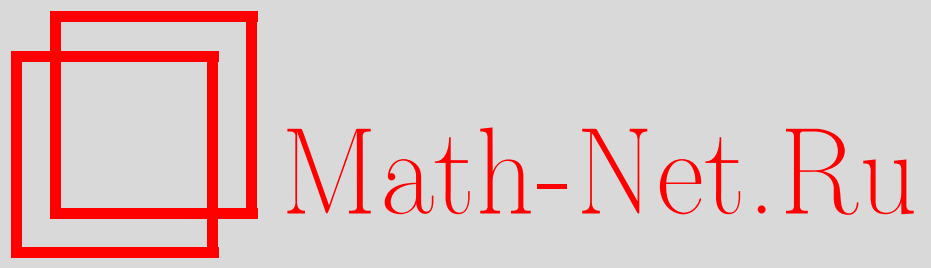

Общероссийский математический портал

Р. К. Ахунжанов, Об анормальных числах, Матем. заметки, 2002, том 72, выпуск 1, 150-152

DOI: https://doi.org/10.4213/mzm659

Использование Общероссийского математического портала Math-Net.Ru подразумевает, что вы прочитали и согласны с пользовательским соглашением http://www.mathnet.ru/rus/agreement

Параметры загрузки:

IP : 54.209 .52 .79

26 апреля 2023 г., 16:11:01 


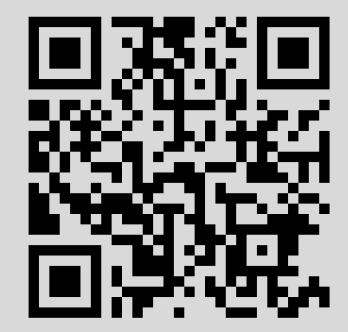




\section{ОБ АНОРМАЛЬНЫХ ЧИСЛАХ}

\section{Р. К. Ахунжанов}

1. Введение. В работе [1] В. Шмидт показал, что каждое из множеств $\mathscr{R}_{q}$ чисел, не являющихся нормальными по натуральному основанию $q \geqslant 2$, является $\alpha$-вьшгрьшным и, следовательно, их пересечение $\bigcap_{q \geqslant 2} \mathscr{R}_{q}$ несчетно. В своей предыдущей работе [2] он доказал, что если натуральньй ряд разбить на два множества $\mathbb{N} \backslash\{1\}=A \sqcup B$ таким образом, что для любых $a \in A, b \in B$ выполнено $\ln a / \ln b \notin \mathbb{Q}$, то найдется несчетное множество чисел $x$, которые будут нормальны по всем основаниям из $A$ и не будут нормальны ни по одному основанию из $B$. С другой стороны, в последнее время вновь стал проявляться интерес к конструкциям анормальных чисел. Так, числа, не являющиеся нормальными ни по какому основанию, были построены в [3] в виде специальных рядов. В настоящей заметке мы даем количественный вариант теоремы Шмидта.

Теорема. Найдется положительная абсолютная әффективная постоянная $\varkappa>0$, обладающал следующим свойством: существует несчетное мнохсество вещественных чисел $x$ таких, что для любого иелого $q \geqslant 2$ и для любого челого неотричательного $n$ выполняется

$$
\left\{q^{n} x\right\} \geqslant \exp \left(-\varkappa q\left(\frac{\ln \bar{q}}{\ln \ln \bar{q}}\right)^{3}\right) .
$$

(Здесь $\{\cdot\}$ означает дробную долю, $a \bar{q}=\max \{q, 3\}$.)

Отметим, что правая часть из неравенства нашей теоремы не зависит от $\boldsymbol{n}$.

Доказательство теоремы проводится по общей схеме, предложенной в [1] (см. также [4, гл. 3]).

2. О выигрышных множествах. В этом пункте мы переформулируем нужным нам образом некоторые резултаты Шмидта из [1], [4].

Пусть $0<\alpha<1,0<\beta<1$ и $\rho>0$. Пусть на вещественной оси отмечено некоторое множество точек $S \subseteq \mathbb{R}$.

Рассматривается игра, в которую играют черные и белые. Сначала черные выбирают на действительной оси замкнутый отрезок $B_{1}$ длины $l\left(B_{1}\right)=2 \rho$. Затем белые выбирают замкнутый отрезок $W_{1} \subset B_{1}$ длины $l\left(W_{1}\right)=\alpha l\left(B_{1}\right)$. Потом черные выбирают замкнутый отрезок $B_{2} \subset W_{1}$ длины $l\left(B_{2}\right)=\beta l\left(W_{1}\right)$ и т.д. Таким способом порождается последовательность вложенных замкнутых отрезков $B_{1} \supset W_{1} \supset B_{2} \supset W_{2} \supset \cdots$ с длинами $l\left(B_{i}\right)=2(\alpha \beta)^{i-1} \rho$ и $l\left(W_{i}\right)=2 \alpha(\alpha \beta)^{i-1} \rho$ $(i=1,2, \ldots)$. Очевидно, что множество $\bigcap_{i=1}^{\infty} B_{i}=\bigcap_{i=1}^{\infty} W_{i}$ состоит из одной единственной точки. Если $\bigcap_{i=1}^{\infty} B_{i} \in S$, то говорят, что белые выиграли игру. 
Далее, множество $S$ назовем $(\alpha, \beta, \rho)$-выигрышным, если белые могут выпграть игру независимо от того, как играют черные.

Лемма 1. Пусть $0<\alpha<1,0<\beta<1, \gamma=1+\alpha \beta-2 \alpha>0, \rho>0$, u nyсть $t \in \mathbb{N}$ maкoe, что $(\alpha \beta)^{t}<\gamma / 2$. Пусть шар $B_{k}=\left(b_{k}, \rho_{k}\right)$ получается в $(\alpha, \beta, \rho)$ игре. Тогда белье могут играть так, что при любой игре черных шар $B_{k+t}$ лежит в полупрямой $x>b_{k}+\rho_{k} \gamma / 2$.

ЗАМЕчАНИЕ. $\gamma<1$.

Обозначим символом $N(g)$ множество чисел, которые в $g$-ичной системе счисления записываются без нулей после запятой.

Лемма 2. Пусть $0<\alpha<1,0<\beta<1, \gamma=1+\alpha \beta-2 \alpha>0, \rho \geqslant \alpha \beta / 2, g \geqslant 3 /(\alpha \beta \gamma)$. Тогда мнохество $N(g)$ является $(\alpha, \beta, \rho)$-выигрышным.

Леммы 1 и 2 являются модификациями аналогичных утверждений из [1]. Их доказательства проводятся по аналогии с проведенными в этой работе. Сначала надо доказать лемму 1 , а затем с ее помощью получить лемму 2.

Пусть натуральный ряд $\mathbb{N}$ представлен в виде дизъюнктного объединения $\mathbb{N}=\sqcup_{j=1}^{\infty} P_{j}$ ариффметических прогрессий $P_{j}$ с первьм членом $m_{j}$ и разностью $d_{j}$. Пусть $\alpha$ и $\beta$ удовлетворяют условиям

$$
1+\alpha \beta-2 \beta>0, \quad 1-2 \alpha \geqslant 0 .
$$

Определим последовательно для всех $j \in \mathbb{N}$ следующие величины:

$$
\alpha_{j}=\alpha, \quad \beta_{j}=\beta(\alpha \beta)^{d_{j}-1}, \quad \gamma_{j}=1+\alpha_{j} \beta_{j}-2 \alpha_{j}, \quad \rho_{j}=(\alpha \beta)^{m_{j}-1} .
$$

Лемма 3. Пусть $0<\alpha<1,0<\beta<1,1+\alpha \beta-2 \beta>0$, и пусть множество $S$ является $(\alpha, \beta, 1)$-выигрышным. Тогда мнохсество $S$ несчетно.

Лемма 4. Пусть множества $N_{j}\left(\alpha_{j}, \beta_{j}, \rho_{j}\right)$-выигрышны. Тогда $\bigcap_{j=1}^{\infty} N_{j}(\alpha, \beta, 1)$-выигрышно.

Лемма 4 фактически является уточнением результата Шмидта о том, что пересечение $\alpha$-вьигрьшшных множеств само является $\alpha$-выигрьшньгм [1], [4].

\section{3. О разбиении натурального ряда на арифметические прогрессии.}

Лемма 5. Натуральный ряд $\mathbb{N}$ можно представить в виде дизвюнктного обвединения $\mathbb{N}=\sqcup_{j=1}^{\infty} P_{j}$ арифметических прогрессий $P_{j}$ с первым членом $m_{j}$ и разностью $d_{j}$ таких, чmo

$$
m_{j} \leqslant d_{j} \leqslant \varkappa_{1} j\left(\frac{\ln \bar{j}}{\ln \ln \bar{j}}\right)^{3}
$$

с некоторой положительной постоянной $\varkappa_{1}$. (Здесь $\bar{j}=\max \{j, 3\}$.)

ДоКАЗАТЕЛЬСтво. Для разбиения натурального ряда на прогрессии нам понадобится ввести факториальную систему счисления. Запись $\overline{a_{n} a_{n-1} \ldots a_{2} a_{1}}$ будет означать сумму $\sum_{k=1}^{n} a_{k} k !$; запись всякого числа в этой системе счисления будет единственной, если цифры $a_{k}$ ограничить условием $0 \leqslant a_{k} \leqslant k$. Единственность разложения следует из равенства $1 \cdot 1 !+2 \cdot 2 !+3 \cdot 3 !+\cdots+n \cdot n !=$ $(n+1) !-1$. Положим $A_{0}=2 \mathbb{N}$ - множество четных натуральных чисел. Пусть число $N=$ $\overline{a_{n} a_{n-1} \ldots a_{2} a_{1}}$ в своей записи не имеет нулей. Сопоставим ему прогрессию $A_{N}=\{x \in \mathbb{N} \mid x=N$ $\bmod (n+2) !\}$. Ее членами являются все числа, у которых первые $n$ цифр совпадают с цифрами в $N$, а $(n+1)$-я цифра - нуль. Отсюда следует, что все эти прогрессии не пересекаются. Очевидно также, что все так определенные прогрессии $A_{N}$, включая $A_{0}$, покрьвают весь натуральньй ряд. (В самом деле, если $x$ нечетно, сохраним все его цифры до первого нуля, остальное откинем. Полученная запись представляет некоторое число $N \in \mathbb{N}$. Согласно определению $x \in A_{N}$.)

Пусть $m_{1}, m_{2}, m_{3}, \ldots$ - начальные члены этих прогрессий, упорядоченные по возрастанию, a $d_{1}, d_{2}, d_{3}, \ldots$ - разности соответствующих прогрессий. Заметим, что всего $n$-значных чисел без нулей равно $n$ !. Следовательно, количество чисел, менњших $k$ ! и без нулей, будет равно $0 !+1 !+2 !+$ $\cdots+(k-1) !$.

Пусть $0 !+1 !+2 !+\cdots+(k-1) !<j \leqslant 0 !+1 !+2 !+\cdots+(k-1) !+k !$. Тогда арифметическая последовательность с таким номером имеет $k$-значный начальный член и, значит, $m_{j} \leqslant(k+1)$ ! $<(k+2)$ ! $=d_{j}$. Так как $(k-1) !<j$, то $d_{j}=(k+2) !<\varkappa_{2} j k^{3}<\varkappa_{1} j(\ln \bar{j} / \ln \ln \bar{j})^{3}$. 
4. Доказательство теоремы. Пусть $\alpha=\beta=1 / 2, m_{j}, d_{j}$-из леммы 5 и $\alpha_{j}, \beta_{j}, \rho_{j}$ выбраны согласно (1). Определим $q_{j}, s_{j}, g_{j}$ из соотношений

$$
q_{j}=j+1, \quad \frac{3}{\alpha_{j} \beta_{j} \gamma_{j}} \leqslant q_{j}^{s_{j}}<\frac{3 q_{j}}{\alpha_{j} \beta_{j} \gamma_{j}}, \quad g_{j}=q_{j}^{s_{j}},
$$

и покажем, что существует несчетное множество точек $x \in \mathbb{R}$, для которых выполняется

$$
\forall n, j \in \mathbb{N}\left\{q_{j}^{n} x\right\} \geqslant q_{j}^{-2 s_{j}} .
$$

Действительно, из соотношений (2) и леммы 5 следует, что выполняются условия леммы 2 , а значит, $N\left(g_{j}\right)$ является $\left(\alpha_{j}, \beta_{j}, \rho_{j}\right)$-выигрышньм. Из соотношений $(1)$ и леммы 4 следует, что пересечение всех множеств $N\left(g_{j}\right)$ является $(\alpha, \beta, 1)$-выигрышным. А из соотношений $(0)$ и леммы 3 следует, что это множество несчетно. Кроме того, оно состоит из тех $x \in \mathbb{R}, g_{j}$-ичная запись которых не содержит цифру 0 после запятой, т.е. для всех его точек $x$ выполнено $\left\{g_{j}^{n} x\right\} \geqslant g_{j}^{-1}$. Выражая $g_{j}$ через $q_{j}$, эту оценку можно записать так: $\left\{q_{j}^{s_{j} n} x\right\} \geqslant q_{j}^{-s_{j}}$. Отсюда легко видеть, что в $q_{j}$-ичной записи числа $x$ не может стоять $2 s_{j}$ нулей подряд, т.е. $\left\{q_{j}^{n} x\right\} \geqslant q_{j}^{-2 s_{j}}$, и тем самым (3) доказано.

Подставляя в (3) оценку для $q_{j}^{s_{j}}$ из (2) и учитывая определения величин $\alpha_{j}, \beta_{j}, \gamma_{j}$ (соотношения (1)) и неравенства леммы 5 , получаем утверждение теоремы.

Теорема доказана.

\section{СПИСОК ЦИТИРОВАННОЙ ЛИТЕРАТУРЫ}

1. Schmidt W. M. // Trans. Amer. Math. Soc. 1966. V. 123. P. 178-199. 2. Schmidt W. M. // Acta. Arith. 1962. V. 7. P. 299-309. 3. Коробов А. Н. // Диофантовы приближения. Математические записки. Т. 2. М., 1996. С. 74-76. 4. Шмидт В. М. Диофантовы приближения. М.: Мир, 1983.

Московский государственный университет им. М.В. Ломоносова 